\title{
Synergistic assembly of hyperbranched polyethylenimine and fatty acids leading to unusual supramolecular nanocapsules $\dagger$
}

\author{
Yu Chen, ${ }^{a}$ Zhong Shen, ${ }^{a}$ Holger Frey, ${ }^{a}$ Julia Pérez-Prieto ${ }^{b}$ and Salah-Eddine Stiriba*b \\ Received (in Cambridge, UK) 15th September 2004, Accepted 11th October 2004 \\ First published as an Advance Article on the web 16th December 2004 \\ DOI: $10.1039 / b 414046 j$
}

Self-assembly of hyperbranched polyethylenimine (PEI) and fatty acids leads to supramolecular inverted micellar structures that are able to irreversibly transfer water-soluble guest molecules into organic solvents.

Modern techniques of liquid-liquid phase transfer involve the use of polymeric supports either as solid phase or dissolved in solution. In contrast to solid-phase concepts, soluble polymeric supports permit reactions to be performed under homogeneous conditions. ${ }^{1}$ In addition, the presence of a polymer support in an organic solvent increases to a large extent the sequestration of a compound (i.e. the solute) from an immiscible solvent such as aqueous media, into the organic solvent, providing a new confined environment. ${ }^{2}$ This is an important point for further specific application in catalysis and drug delivery. ${ }^{3}$ In this context, structurally precise dendrimers with their highly branched and multifunctional globular structure have been shown to exhibit topological encapsulation of various guests. ${ }^{4}$ Encapsulation can be achieved using the unimolecular micelle approach with core-shell structures to dissolve organic molecules in dendrimers, ${ }^{5}$ or by utilizing the organosoluble alkyl amide-modified dendrimers known as "dendritic box" to extract water soluble guests to organic solvents. ${ }^{6}$ Unfortunately, dendrimer synthesis is usually limited to a laboratory scale due to the stepwise iterative approach. However, the less defined hyperbranched polymers can be prepared conveniently from $\mathrm{AB}_{\mathrm{m}}$ type monomers on a large scale. When using slow monomer addition techniques, hyperbranched polymers with rather narrow polydispersity and dendrimer-like properties are obtained. Hyperbranched polyols prepared in a onestep process, esterified with fatty acid chlorides in a second step, display an amphiphilic core-shell structure. ${ }^{7}$ They have been successfully used to extract and irreversibly encapsulate various polar dyes from the aqueous phase.

Surprisingly, all dendrimer-based core-shell architectures for phase transfer processes have been obtained via covalent linking of hydrophobic alkyl chains to the polyfunctional core, followed by several purification steps. Only one report described a noncovalent concept for organosoluble, inverted micelles, prepared by self-assembly of various generations of amine terminated poly(amidoamine) (PAMAM) dendrimers and fatty acids, relying on acid-base interaction. ${ }^{8}$ For hyperbranched polymers, however, to our knowledge there is no report on supramolecular nanocapsules obtained from non-covalent assembly.

$\dagger$ Electronic supplementary information (ESI) available: IR, UV-vis spectra, table of encapsulation data and pictures of dyes transport. See http://www.rsc.org/suppdata/cc/b4/b414046j/

*salah.stiriba@uv.es
Here we report a convenient one-step process for the preparation of supramolecular nanocapsules by electrostatic interaction between fatty acids and a commercially available hyperbranched polyamine, namely polyethylenimine (PEI) (Scheme 1).

The addition of fatty acids, namely lauric acid (LA) $\mathrm{C}_{11} \mathrm{H}_{23} \mathrm{CO}_{2} \mathrm{H}$ or palmitic acid (PA) $\mathrm{C}_{15} \mathrm{H}_{31} \mathrm{CO}_{2} \mathrm{H}$ to a chloroform solution of hyperbranched polyethylenimine $\mathrm{PEI}_{232}\left(M_{\mathrm{n}}=\right.$ $\left.10,000 \mathrm{~g} \mathrm{~mol}^{-1}, \mathrm{DB}=60 \%\right)^{9}$ in 0.6 (LA/amine) ratio spontaneously afforded the respective polyelectrolyte-surfactant complexes, i.e., LA-PEI $\mathbf{I}_{23}$ and $\mathbf{P A}-\mathbf{P E I}_{\mathbf{2 3 2}}$. This conclusion is

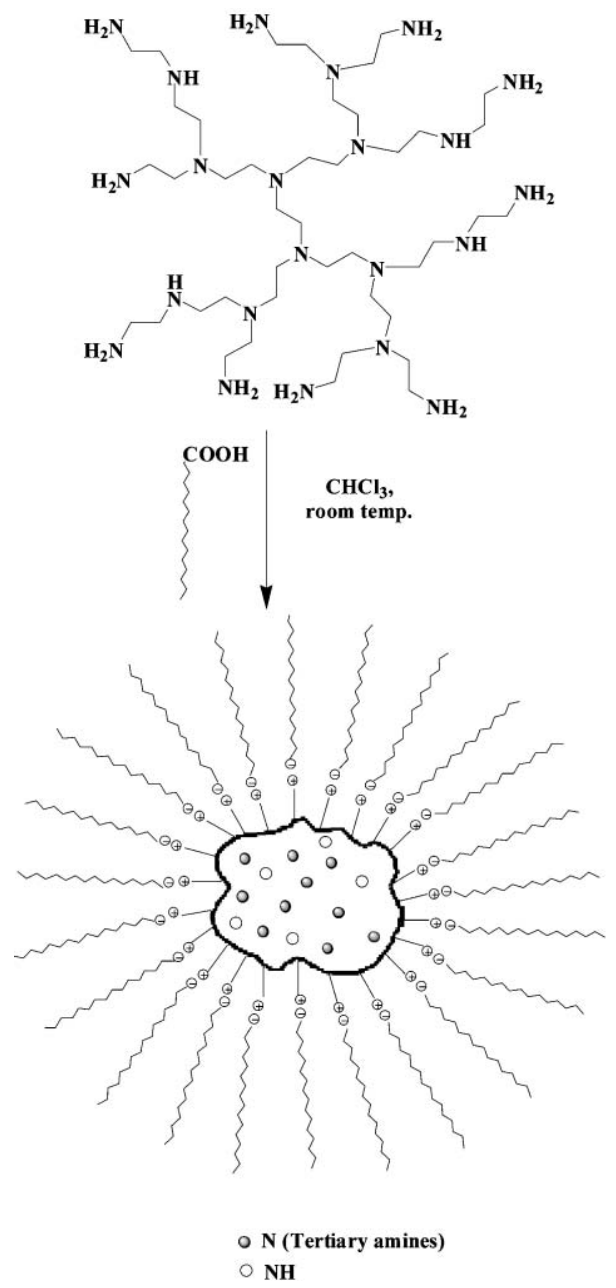

Scheme 1 Schematic illustration of an inverted, supramolecular micelle based on the self-assembly of hyperbranched polyethylenimine and palmitic acid. 
supported by various analytical methods. FT-IR absorbance spectra (Supp. Mater.) $\dagger$ show the disappearance of the characteristic symmetric $(\mathrm{C}=\mathrm{O})$ band of the fatty acids at $1700 \mathrm{~cm}^{-1}$ upon addition to $\mathrm{PEI}_{232}$ in solution, accompanied by the appearance of a sharp, asymmetric band at $1550 \mathrm{~cm}^{-1}(\mathrm{C}=\mathrm{O})$, typical of the carboxylate salt. This demonstrates that the fatty acid molecules are deprotonated and the terminal, primary $\mathrm{NH}_{2}$ groups of PEI (fraction of terminal, T-groups $=33.3 \%, \mathrm{p} K_{\mathrm{a}}=9.64$ ) are protonated first, ${ }^{10}$ leading to ionic interaction and thus a polyelectrolyte-surfactant complex with a rather hydrophilic core and a hydrophobic alkyl periphery that acts as a "supramolecular nanocapsule".

Upon addition of larger amounts of carboxylic acid, the secondary amine groups of the linear units (fraction $\mathrm{L}=40 \%$, $\left.\mathrm{p} K_{\mathrm{a}}=8.59\right)$ are also protonated and interact with the alkyl carboxylates.

Formation of the hyperbranched polyelectrolyte-surfactant complex can be expected to lead to pronounced changes of the conformation of the hyperbranched polymer. This issue was studied by solution viscosity measurements of the mixtures LA$\mathbf{P E I}_{232}$ at different ratios of both components. Interestingly, Fig. 1 shows a systematic decrease in the specific viscosity $\left(\eta_{\text {spec }}\right)$, when increasing the lauric acid proportion at fixed concentration of $\mathrm{PEI}_{232}$. For instance, pure $\mathrm{PEI}_{232}$ displays an intrinsic viscosity $[\eta]=10.6 \mathrm{~mL} \mathrm{~g}^{-1}$, while a mixture of $\mathrm{LA}$ and $\mathrm{PEI}_{232}$ at LA/amine ratio of 0.6 shows clearly reduced intrinsic viscosity $[\eta]=6.5 \mathrm{~mL} \mathrm{~g}^{-1}$ (both extrapolated to $c=0 \mathrm{~g} \mathrm{~L}^{-1}$ ). We attribute this pronounced difference to a very compact solution structure, due to the supramolecular nanocapsules LA-PEI 232 and PA$\mathbf{P E I}_{232}$ formed in apolar solvents. A comparative study with the linear analogue $\mathrm{LPEI}_{348}$ assembled with $\mathrm{LA}$ in $\mathrm{LA} /$ amine ratio of 0.6 also provided a polyelectrolyte complex (FTIR), LA$\mathbf{L P E I}_{348}$, however, no similarly compact structure as the hyperbranched $\mathrm{PEI}_{232}$. This is evident from the values of the specific and particularly the intrinsic viscosity of $\mathbf{L A}-\mathbf{L P E I}_{\mathbf{3 4 8}}$, $[\eta]=13.1 \mathrm{~mL} \mathrm{~g}^{-1} \cdot 11$

Dynamic Light Scattering (DLS) showed the average size of PEI $_{232}\left(M_{\mathrm{n}}=10^{4} \mathrm{~g} \mathrm{~mol}^{-1}\right)$ to be $5.1 \mathrm{~nm}$ at $c=10^{-4} \mathrm{M}$, whereas

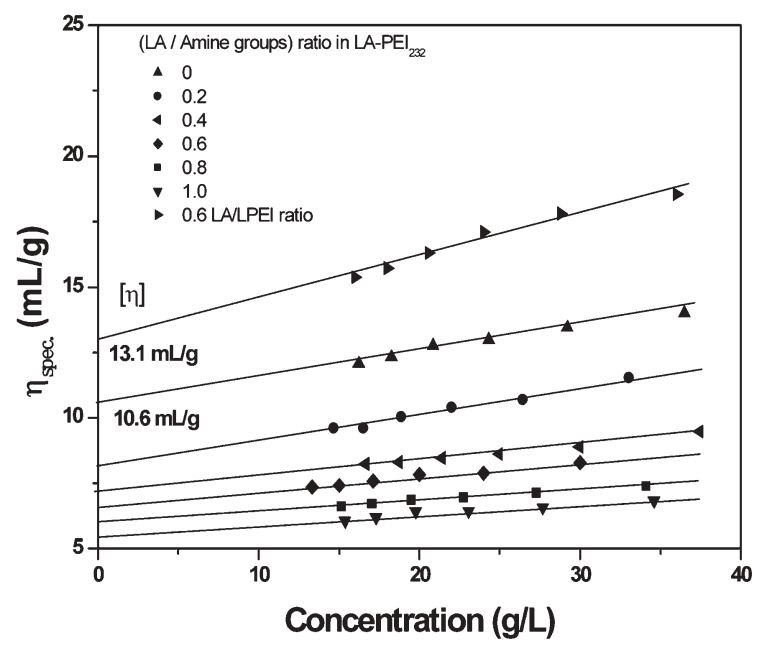

Fig. 1 Viscosity measurements of $\mathbf{L A}-\mathbf{P E I}_{\mathbf{2 3 2}}$ mixtures in chloroform at different ratios of the components. Data for a linear analogue $\mathbf{L A}-\mathbf{P E I}_{\mathbf{2 3 2}}$ are also shown for comparison. the size of associated $\mathbf{L A}-\mathbf{P E I}_{\mathbf{2 3 2}}$ (LA/amine groups in PEI $=60 \%$ ) was $6.5 \mathrm{~nm}$. These DLS values also support the conclusion that amphiphilic hyperbranched polymers with a hydrophilic core and a hydrophobic shell form stable "supramolecular nanocapsules". ${ }^{2}$

Recently, PEI has been covalently modified via amidation at elevated temperatures with palmitic acid, followed by a tedious purification process to remove acidic traces as well as by imine condensation into ketones and aldehydes. Encapsulation of both silver nanoparticles and dyes was reported. ${ }^{13}$ The present supramolecular nanocapsules possess several advantages over the covalent analogues: $i$ ) convenient preparation without need for further purification; ii) facile variation of the composition to finetune the molecular nanocapsules as a function of the acid-PEI ratios.

In order to demonstrate the phase-transfer properties of the nanocapsules, two polar dyes, i.e., Congo Red (CR) and Methyl Orange (MO) were dissolved in water (Supp. Mater.). The aqueous phases were extracted with chloroform solutions containing either $\mathbf{P A}-\mathbf{P E I}_{\mathbf{2 3 2}}$ or $\mathbf{L A}-\mathbf{P E I}_{\mathbf{2 3 2}}$. Fig. 2 shows an increase in the absorbance of Methyl Orange in the chloroform phase $\left(\lambda_{\max }=424 \mathrm{~nm}\right)$, indicating dynamic transport of the dye from the aqueous phase $\left(\lambda_{\max }=464 \mathrm{~nm}\right)$ into the organic medium. It should be emphasized that neither of the two components alone showed a similar phase transfer effect, supporting the synergistic behavior of the system. The number of encapsulated dye molecules was found to be 8.5 and 8.6 for $\mathbf{L A}-\mathbf{P E I}_{232}$ and $\mathbf{P A}-\mathbf{P E I} \mathbf{I}_{232}$ respectively, independent of the length of the alkyl chain of the fatty acid used. Fewer CR dye molecules were encapsulated (3.3 and 2.9 dyes in $\mathbf{L A}-\mathbf{P E I}_{\mathbf{2 3 2}}$ and $\mathbf{P A}-\mathbf{P E I}_{\mathbf{2 3 2}}$, respectively). This is tentatively explained by the large size of CR. The number of encapsulated dye molecules was verified to be PEI core molecular weight dependent, as demonstrated using the larger $\mathbf{P E I}_{\mathbf{5 8 1}}$ $\left(M_{\mathrm{n}}=25000 \mathrm{~g} \mathrm{~mol}^{-1}\right)$ (Table 1, Supp. Mater.). Interestingly, in the latter case $\mathbf{L A}-\mathbf{P E I}_{\mathbf{5 8 1}}$ showed high encapsulation capacity for CR (7.2) whereas the same $\mathbf{P E I}_{\mathbf{5 8 1}}$ covalently modified with a long alkyl chain was found to encapsulate only a few CR dye molecules (0.2) in a previous study. ${ }^{13 b}$ The hyperbranched architecture of PEI was crucial to obtain dye extraction, as indicated by the inability of the linear analogue $\mathbf{L A}-\mathbf{L P E I}_{\mathbf{3 4 8}}$ to encapsulate $\mathrm{CR}$ and its

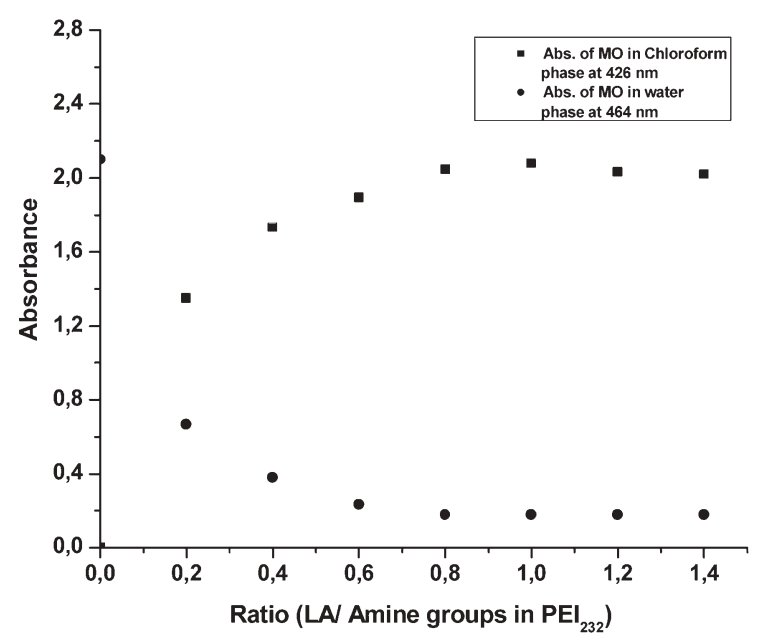

Fig. 2 Absorbance of Methyl Orange (MO) in the chloroform layer containing inverted micelle $\mathbf{L A}-\mathbf{P E I}_{\mathbf{2 3 2}}$ and its traces in the aqueous layer. 
insignificant extraction of MO dyes as well. Upon addition of acid $(\mathrm{pH}=2)$ to the organic phase containing the encapsulated dyes, release of the dyes into the aqueous layer was observed, supporting solubilization by supramolecular nanocapsules.

In conclusion, the data demonstrate that supramolecular selfassembly of a well known hyperbranched polymer with fatty acids leads to unusual nanocapsules. The synergy between both components affords phase transfer properties due to the polyelectrolyte-surfactant complexes.

S.-E.S. thanks the Ministerio de Educación y Ciencia (Spain) for support in the context of the "Ramón y Cajal" national program. $\mathrm{He}$ is also indebted to the Alexander von Humboldt Foundation for support. We thank Mrs. X. Yuan (U. Mainz) for assistance with DLS measurements.

Yu Chen, ${ }^{a}$ Zhong Shen, ${ }^{a}$ Holger Frey, ${ }^{a}$ Julia Pérez-Prieto ${ }^{b}$ and Salah-Eddine Stiriba*b

${ }^{a}$ Institut für Organische Chemie, Johannes Gutenberg-Universität, Duesbergweg 10-14, 55099, Mainz, Germany.

E-mail: hfrey@mail.uni-mainz.de; Fax: (+49) 6131-39 26106;

Tel: (+49) 6131-3926106

${ }^{b}$ Instituto de Ciencia Molecular/Universidad de Valencia, Av. Vicent Andrés Estelles, S/N, 46100, Burjassot, Valencia, Spain.

E-mail: salah.stiriba@uv.es; Fax: (+34) 96-354 4939;

Tel: (+34) 96-3543052

\section{Notes and references}

1 C. C. Tzschucke, C. Markert, W. Bannwarth, S. Roller, A. Hebel and R. Haag, Angew. Chem., Int. Ed., 2002, 41, 3964.
2 T. Sekine and Y. Hasegawa, Solvent Extraction Chemistry. Fundamentals and Applications, 1997, Marcel Dekker, New York.

3 For supramolecular drug delivery, see: R. Haag, Angew. Chem., Int. Ed., 2004, 43, 278.

4 G. R. Newkome, C. N. Moorefield and F. Vögtle, Dendritic Molecules: Concepts, Syhthesis, Perspectives, VCH, Weinheim, 2001.

5 (a) G. R. Newkome, C. N. Moorefield, G. R. Baker, M. J. Saunders and S. H. Grossman, Angew. Chem., Int. Ed. Engl., 1991, 30, 1178; (b) M. W. P. L. Baars, R. Kleppinger, M. H. J. Koch, S. L. Yeu and E. W. Meijer, Angew. Chem., Int. Ed., 2000, 39, 1285.

6 S. Stevelmans, J. C. M. van Hest, J. F. G. A. Jansen, D. A. F. J. van Boxtel, E. M. M. de Brabander-van den Berg and E. W. Meijer, J. Am. Chem. Soc., 1996, 118, 7398.

7 S.-E. Stiriba, H. Kautz and H. Frey, J. Am. Chem. Soc., 2002, 124, 9698.

8 V. Chechik, M. Zhao and R. M. Crooks, J. Am. Chem. Soc., 1999, 121, 4910.

9 Polyethylenimines $\mathrm{PEI}_{232}\left(\mathrm{DB}=60 \%, \mathrm{PDI}=2.5, M_{\mathrm{n}}=10000 \mathrm{~g} \mathrm{~mol}^{-1}\right.$ ) and $\mathrm{PEI}_{581}\left(\mathrm{DB}=60 \%\right.$, PDI $\left.=2.5, M_{\mathrm{n}}=25000 \mathrm{~g} \mathrm{~mol}^{-1}\right)$. From inverse gated ${ }^{13} \mathrm{C}$ NMR in $\mathrm{CDCl}_{3}$ integration, $\mathrm{PEI}_{232}$ possesses $33.3 \%$ $\mathrm{NH}_{2} ; 40 \% \mathrm{NH}$ and $26.7 \%$ tertiary amine). For $\mathrm{PEI}_{581}$ see ref. $13 \mathrm{~b}$.

10 M. Borkovec and G. J. M. Koper, Macromolecules, 1997, 30, 2151.

11 Linear $\mathrm{LPEI}_{348}$ was prepared starting from poly(2-ethyl-2-oxazoline) according to Kem's method: K. M. Kem, J. Polym. Sci., Polym. Chem. Ed., 1979, 17, 1997.

12 The concentration of $\mathbf{L A}-\mathbf{P E I}_{\mathbf{2 3 2}}$ used for the encapsulation was $1.1 \times$ $10^{-5} \mathrm{M}$, which is lower than that used for DLS analysis $\left(10^{-4} \mathrm{M}\right)$. We deduce that the dyes were in fact encapsulated by single micellar structures rather than aggregated ones.

13 (a) C. Aymonier, U. Sclotterbeck, L. Antonietti, P. Zacharias, R. Thomann, J. C. Tiller and S. Mecking, Chem. Commun., 2002, 3018; (b) M. Krämer, J.-F. Stumbé, H. Türk, S. Krause, A. Komp, L. Delineau, S. Prokhorova, H. Kautz and R. Haag, Angew. Chem., Int. Ed., 2002, 41, 4252. 